\title{
The impact of keratoconus treatment with the Athens Protocol (partial topography-guided photorefractive keratectomy combined with higher-fluence corneal collagen cross-linking) on quality of life: a long-term study
}

This article was published in the following Dove Medical Press journal: Clinical Ophthalmology

Anastasios John

Kanellopoulos ${ }^{1,2}$

'Department of Ophthalmology, Laservision.gr, Clinical and Research Eye Institute, Athens, Greece; ${ }^{2} \mathrm{NYU}$ Medical School, Department of Ophthalmology, New York, NY, USA

Video abstract

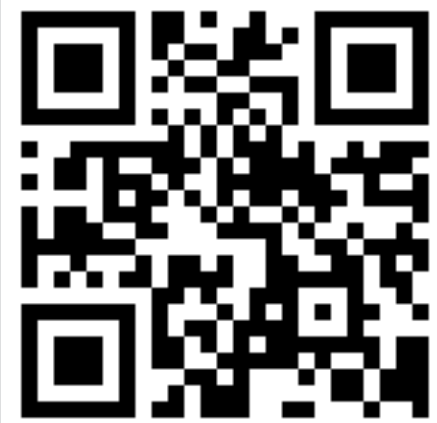

Point your SmartPhone at the code above. If you have a QR code reader the video abstract will appear. Or use:

http://youtu.be/QWQUwgbe 88 E

Correspondence: Anastasios John Kanellopoulos

Laservision.gr Clinical and Research

Eye Institute, 17 Tsocha Street,

Athens II52I, Greece

Tel +302107472777

Fax +30 21 07472789

Email ajk@brilliantvision.com
Purpose: The aim of this study was to evaluate the health-related quality of life (HRQoL) before and after the management of keratoconus $(\mathrm{KCN})$ with Combined Topography-Guided Transepithelial partial photorefractive keratectomy (PRK) and corneal Cross-Linking: the Athens Protocol.

Patients and methods: Thirty-four consecutive patients treated for progressive KCN were selected for retrospective telephone interview and individual questionnaire submission. A disease-specific HRQoL questionnaire was administered before and after the Athens Protocol intervention, using the following administrative questionnaires: National Eye Institute Visual Function Questionnaire (NEI VFQ) 25/39 and IVI-28. The disease grade was ranked according to the Amsler-Krumeich classification (KCN severity). Reliability of collected data was evaluated with Cronbach's $\alpha$ test.

Results: Patients' age ranged from 15 to 51 years at the time of the operation. Mean time before the operation during which the questionnaires were administered was $52 \pm 6$ months. General visual acuity, psychological well-being, and driving ability showed significant improvement. Younger keratoconic patients tend to have more enhanced psychological status and to cope better with difficulties in everyday activities (prior to operation) than older patients.

Conclusion: The Athens Protocol treatment has been proven to be beneficial to patients in a large number of aspects.

Keywords: KCN, Athens Protocol, topography-guided PRK, quality of life, HRQoL questionnaire, NEI VFQ, IVI-28

\section{Introduction}

The concept of quality of life (QoL), which seems to have recently gained increasing popularity, is not new. Aristotle was the first among those who defined various such concepts and tried to connect them to values of life, such as prosperity and a general notion of "well-being". QoL research interest in social sciences and psychology re-emerged in the 1940s. After the economic boom that followed the war and the progress of medicine in the 1960s, the concept of prosperity and the need for a better QoL, and not just an extension of the life cycle, became the center of interest. ${ }^{1}$ Today, QoL may be defined as a multidimensional assessment of the current living conditions of the individual within the culture and value framework in which he/she lives. ${ }^{2}$ Furthermore, it is a set 
of general well-being measures that combines the objective indicators and subjective assessments of natural, physical, social, and emotional well-being taking into consideration the extent of personal growth and meaningful activities. ${ }^{3}$

Health-related quality of life (HR-QoL) reflects the difference between patients' expectations and the existing state of their health, which may undergo changes, in line with the progression of their disease. ${ }^{4}$ States often consider HR-QoL data when deciding on the allocation of limited financial resources for health. ${ }^{5}$ HR-QoL is evaluated from data derived mainly from weighted questionnaires. The questions may have only one answer or a certain range and may involve either one or more aspects of health status (multidimensional). ${ }^{6}$

Individual questionnaire completion can be achieved electronically, via mail or phone interview, or by presenting the questionnaire directly to the respondent. ${ }^{7}$ Although the postage-mail method can cover large geographical areas, it assures enough time for completion and comes with a minimum cost; however, it has the lowest responsiveness compared to other methods. ${ }^{8}$ The electronic individual filling (interview via computer) method is objective, has access to a large population, and provides an automated flow of questions; however, it requires some degree of computer literacy and obviously access to the Internet. HR-QoL evaluation questionnaires must satisfy certain criteria to provide clinically useful results, such as validity (content, criterion, and construct), reliability, sensitivity, and responsiveness. ${ }^{9}$

At the time of this study, there was no similar questionnaire for keratoconus $(\mathrm{KCN}) .{ }^{10}$ The National Eye Institute Visual Function Questionnaire (NEI VFQ) was originally developed for the evaluation of QoL in relation to vision diseases such as age-related macular degeneration, but has also been used for assessing the $\mathrm{KCN}$ effect on visionspecific QoL (the Collaborative Longitudinal Evaluation of Keratoconus [CLEK] study). ${ }^{11}$ The Impact of Vision Impairment (IVI) questionnaire, which aims to assess the restriction of participation in daily living as well as the effectiveness of rehabilitation outcomes in low vision, has also been used by other research groups on $\mathrm{KCN}$ in the peer-review literature: Gothwal et $\mathrm{al}^{12}$ employed the IVI questionnaire and few years later Khadka et al ${ }^{13}$ employed the Keratoconus Outcomes Research (KOR) questionnaire for the assessment of KCN only; Labiris et al ${ }^{14}$ employed the VFQ questionnaire for the assessment of $\mathrm{KCN}$ and a combined laser-corneal collagen cross-linking (CXL) treatment. However, QoL is not the same as Quality of Vision (QoV). The NEI-VFQ has been used to assess visual functioning, which is only one domain in the assessment of QoL. Our study links the Athens Protocol intervention to improvements in the QoL. More extensive studies may be helpful in offering quantitative data on QoVand QoL-specific improvements.

The aim of the present study is to evaluate the selfreported improvement of $\mathrm{HR}-\mathrm{Q}$ LL in the $\mathrm{KCN}$ patients treated with CXL and specifically the Athens Protocol procedure.

\section{Patients and methods}

The survey was conducted in Laservision Clinical and Research Eye Institute, Athens, Greece. Patients were a consecutive case series and had been previously subjected to the Athens Protocol procedure for the management of $\mathrm{KCN}$ to either eye. A minimum sample size of 34 patients was established based on the estimates of type I error $\alpha=0.05$. Twenty of the patients (58.8\%) underwent bilateral Athens Protocol procedure and the rest $(41.2 \%)$ underwent unilateral. In the latter situation, the treated eye was based on the predicted QoL improvement; it could be either the dominant or the non-dominant one. Two VFQs were employed as follows: the NEI VFQ 25 and 39, ${ }^{15,16}$ for which an official Greek version is available (freely available from the RAND Organization), ${ }^{17,18}$ and the Impact of Vision Impairment IVI-28, ${ }^{19,20}$ which required translation. Permission to translate the IVI into Greek language was obtained from their authors, along with license to use it for this purpose. The IVI questionnaire has been designed by the Centre for Ophthalmic Research Australia (CERA) to assess the restriction of participation in daily living as well as the effectiveness of rehabilitation outcomes in low vision, unlike most vision-specific questionnaires that typically assess visual functioning. IVI's raw scores were converted into Rasch person measures following the developers' instructions. ${ }^{21}$ The scores from the questionnaires were measured on a scale from 0 to 100 , where 100 determines the optimum level of QoL in relation to vision.

Patients initially completed all questionnaires onsite during the pre-operative screening, whereas the post-operative version was filled via phone interview, by the author himself, at 4 years following the procedure.

The study received approval by the ethics committee of our Institution (Laservision Clinical and Research Eye Institute) and adhered to the tenets of the Declaration of Helsinki, which includes the ethical principles of medical research for the protection of personal data of the participants. Informed consent was provided and documented in a written form from each patient prior to the time of the observation. 
Inclusion criteria included the following: diagnosis of progressive $\mathrm{KCN}$, documentation after a complete ophthalmological examination, and subsequent treatment with the Athens Protocol procedure.

All patients studied were established as contact lens intolerant with rigid gas permeable contacts and some had experience with soft contact lenses in previous evaluations. All patients evaluated and treated had been wearing spectacles and prior to considering surgery were given updated refractions and had been made to wear new updated spectacles. Post-operatively all patients were evaluated with either no spectacles, with appropriate spectacles if needed, and with soft contact lenses to those who preferred them to spectacles.

$\mathrm{KCN}$ severity prior to the operation was established through information collected from patient files, according to the Amsler-Krumeich classification. ${ }^{22}$ The eyes were classified into two categories according to their pre-operative corrected distance visual acuity (CDVA) with Snellen charts, which were converted to a logarithm of the minimal angle of resolution (logMAR) for statistical analysis. These two categories were created to distinguish, especially in cases of bilateral $\mathrm{KCN}$, the eyes with the worst visual acuity. In cases of unilateral $\mathrm{KCN}$, the keratoconic eye was the one with the worst visual acuity. Age was an additional parameter evaluated by Spearman testing (parameters did not follow normal distribution). Gender, level of education, and use of spectacles or contact lenses were also evaluated, by median value comparison (with the null hypothesis that they do not affect the values of the questionnaires), considering their possible influence on the responses derived. The questionnaire reliability was tested with the Shapiro-Wilk W test and Cronbach's $\alpha$ criterion (to check normality of data). Data were then evaluated with non-parametric analysis.

The extent to which scores were influenced by the descriptive elements of the sample (such as age, gender, and level of education) pre- and post-operatively was then evaluated. The difference in the response scores (postoperative to pre-operative) in each question was considered. Statistical analysis was performed by SPSS version 22 (IBM Corporation., Armonk, NY, USA). $p$-values less than 0.05 were indicative of statistically significant differences.

\section{Results}

\section{Descriptive statistics of sample population}

A total of 34 patients ( 25 males, 9 females) participated in this study. Patient age ranged from 15 to 51 years at the time of the operation (mean $=29.5$ years) (Table 1 ). Most of the patients had bilateral surgery: 54 of the 59 keratoconic eyes
Table I Descriptive statistics of sample population

\begin{tabular}{|c|c|c|}
\hline & Pre-operative & Post-operative \\
\hline \multicolumn{3}{|l|}{ Gender, ${ }^{\mathrm{a}} \mathrm{n}(\%)$} \\
\hline Male & $25(73.5)$ & \\
\hline Female & $9(26.5)$ & \\
\hline \multicolumn{3}{|c|}{ Educational level, ${ }^{a}$ n (\%) } \\
\hline Secondary & II (32.4) & \\
\hline Higher & $23(67.6)$ & \\
\hline \multicolumn{3}{|c|}{ Visual acuity ${ }^{\mathrm{b}}$ (mean $\left.\pm \mathrm{SD}\right)$} \\
\hline Higher & $0.49 \pm 0.9 \log M A R$ & $0.07 \pm 0.09 \log M A R$ \\
\hline Lowest & $1.05 \pm 1.15 \log M A R$ & $0.16 \pm 0.12 \log M A R$ \\
\hline \multicolumn{3}{|c|}{ Keratoconus staging, ${ }^{c} \mathrm{n}(\%)$} \\
\hline I & $9(26.5)$ & \\
\hline 2 & $13(38.2)$ & \\
\hline 3 & $12(35.3)$ & \\
\hline \multicolumn{3}{|c|}{ Spectacles/contact lens use, ${ }^{d} \mathrm{n}(\%)$} \\
\hline None & $17(50)$ & $19(55.9)$ \\
\hline At least one eye & $5(14.7)$ & $4(11.7)$ \\
\hline Both eyes & $12(35.3)$ & II (32.4) \\
\hline
\end{tabular}

Notes: a'Gender and educational level same for pre- and post-operative. ${ }^{\mathrm{V}}$ isual acuity refers to corrected distance visual acuity. 'Keratoconus staging, as determined prior to the operation. ${ }^{d}$ Refers to post-operative.

were subjected to the Athens Protocol. Post-operative QOL assessment and questionnaires were completed at 4 years following the procedure.

\section{Normality check}

Normality of the data (reply scores) was tested by using the Shapiro-Wilk W test (Table 2). Most scores do not follow the normal distribution $(p<0.05)$. Pre-operatively, the scores of distant vision activities, mental health, and driving ability have a normal distribution in VFQ 25, whereas in VFQ 39 (Table 2A), we identified only numerical changes in distant vision activities and mental health scores. Differences in the statistical significance between the two VFQ NEI questionnaires for general vision status were noted.

The IVI-28 scores (Table 2B) differ considerably as to their statistical significance. Mobility and independence both before and after surgery have a normal distribution, as opposed to reading and information awareness that maintained the same statistically significant value. The scale of emotional well-being has normal distribution only post-operatively.

\section{Non-parametric median comparisons}

Since most scores did not satisfy the conditions of normality, all the following comparisons are non-parametric. Therefore, we evaluate median differences (MDs) before and after surgery. The results are summarized in Table $3 \mathrm{~A}-\mathrm{C}$. 
Table 2 Normality check results (Shapiro-Wilk W test) for NEI VFQ 25 and 39 and IVI-28

\begin{tabular}{|c|c|c|c|c|c|c|c|c|}
\hline & \multicolumn{4}{|c|}{ Pre-operative } & \multicolumn{4}{|c|}{ Post-operative } \\
\hline & W stat & $p$ & W stat & $p$ & W stat & $p$ & W stat & $p$ \\
\hline & \multicolumn{2}{|c|}{ NEI VFQ 25} & \multicolumn{2}{|c|}{ NEI VFQ 39} & \multicolumn{2}{|c|}{ NEI VFQ 25} & \multicolumn{2}{|c|}{ NEI VFQ 39} \\
\hline \multicolumn{9}{|l|}{ (A) NEI VFQ 25 and 39} \\
\hline General health status & 0.858 & 0.001 & 0.933 & 0.039 & 0.862 & 0.001 & 0.925 & 0.022 \\
\hline General vision status & 0.867 & 0.010 & 0.969 & 0.434 & 0.689 & 0.001 & 0.869 & 0.001 \\
\hline Ophthalmic pain & 0.897 & 0.004 & 0.897 & 0.004 & 0.787 & 0.001 & 0.787 & 0.001 \\
\hline Near vision activities & 0.924 & 0.021 & 0.838 & 0.002 & 0.807 & 0.001 & 0.718 & 0.001 \\
\hline Distant vision activities & 0.971 & 0.483 & 0.958 & 0.208 & 0.796 & 0.001 & 0.813 & 0.001 \\
\hline Social functionality & 0.764 & 0.001 & 0.753 & 0.001 & 0.621 & 0.001 & 0.613 & 0.001 \\
\hline Mental health & $0.94 I$ & 0.066 & 0.945 & 0.086 & 0.770 & 0.001 & 0.775 & 0.001 \\
\hline Difficulties in activities & 0.902 & 0.005 & 0.911 & 0.009 & 0.653 & 0.001 & 0.675 & 0.001 \\
\hline Dependence & 0.790 & 0.001 & $0.78 \mathrm{I}$ & 0.001 & 0.474 & 0.001 & 0.465 & 0.001 \\
\hline Driving & 0.953 & 0.291 & 0.953 & 0.291 & 0.759 & 0.001 & 0.759 & 0.001 \\
\hline Color vision & 0.172 & 0.001 & 0.172 & 0.001 & 0.172 & 0.001 & 0.172 & 0.001 \\
\hline Peripheral vision & 0.773 & 0.001 & 0.773 & 0.001 & 0.624 & 0.001 & 0.624 & 0.001 \\
\hline \multicolumn{9}{|l|}{ (B) IVI-28 } \\
\hline Mobility and independence & \multicolumn{2}{|c|}{$72.47 \pm 15.49$} & 0.951 & 0.145 & \multicolumn{2}{|c|}{$84.35 \pm 15.36$} & 0.925 & 0.121 \\
\hline Emotional well-being & \multicolumn{2}{|c|}{$63.7 I \pm 12.60$} & 0.942 & 0.078 & \multicolumn{2}{|c|}{$81.57 \pm 14.43$} & 0.890 & 0.016 \\
\hline Reading and information awareness & \multicolumn{2}{|c|}{$59.93 \pm 8.54$} & 0.866 & 0.001 & \multicolumn{2}{|c|}{$76.37 \pm 9.34$} & 0.877 & 0.001 \\
\hline
\end{tabular}

Abbreviations: NEI, National Eye Institute; VFQ, Visual Function Questionnaire.

General health and color vision MDs did not show statistical significance, that is, they do not appear to be affected by $\mathrm{KCN}$ management. For social functionality and peripheral vision, although they appear to have a Wilcoxon statistically significant difference, the Hodges-Lehmann estimator reveals zero value at the lower limit of the CI and thus nonstatistical significance, which refutes the previous audit and reveals that there is no difference in median scores before and after surgery. All other scores indicate a clear difference before and after surgery. Similar results are noted for VFQ 39. Wilcoxon $p$-value for general health is smaller, but this time, the MD shows no statistical significance. All IVI-28 scores show statistical significance. The Hodges-Lehmann estimator verifies the conclusion of the previous check, as long as there is no zero value in $\mathrm{CI}$.

\section{Non-parametric checks for correlations on descriptive score elements}

Age appears to have no correlation with the variables associated with the replies in either questionnaire (Table $4 \mathrm{~A}$ and $\mathrm{B}$ ). Gender affects pre-operative mental health scores $(p=0.033)$, whereas there is no post-operative association. The level of education does not appear to affect pre-operative scores but appears to affect distant vision activities ( $p=0.045$ for VFQ 25 and $p=0.028$ for VFQ 39). No association was noted for the IVI-28. Visual acuity does not appear to affect pre-operative scores in any questionnaire. Post-operatively, there is a positive correlation of visual acuity with mental health score (Spearman's $\rho=0.055$ and $p=0.012$ ). In VFQ 39, visual acuity correlated with general vision status ( $\rho=0.563$ and $p=0.012$ ), near vision activities $(\rho=0.467$ and $p=0.038)$, and mental health ( $\rho=0.509$ and $p=0.022$ ). The scores of the IVI 28 questionnaire did not show any correlation with visual acuity. Disease progression (KCN severity) involves only pre-operative data and there is positive correlation of this with the VFQ 25 and 39 questionnaires ( $p=0.01$ and $p=0.01$, respectively). The IVI-28 is influenced by the stage of the disease on the "Reading and Information Awareness" $(p=0.049)$. The use of spectacle and/or contact lens involves the post-operative stage. Results showed that the scores are not affected by the patients' use of any means of refractive correction in any questionnaire.

\section{Non-parametric checks for correlations on descriptive elements of the post- to pre-operative differences in scores}

There is no statistically significant correlation with age in most cases. Age does not show any correlation with the scores of IVI-28. Positive correlations are noted only from the VFQ 39 as follows:

1. Difficulties in activities and age: correlation coefficient (Spearman) $\rho=0.433(p=0.011)$ pre-operatively and $\rho=0.449(p=0.008)$ post-operatively. 
Table 3 Difference in median of responses post- to pre-operatively

\begin{tabular}{|c|c|c|c|c|c|c|c|c|}
\hline & \multicolumn{2}{|c|}{ Pre-operative } & \multicolumn{3}{|c|}{ Post-operative } & \multicolumn{2}{|l|}{$95 \% \mathrm{Cl}$} & \multirow[t]{2}{*}{$p$-value ${ }^{b}$} \\
\hline & Median & IQR & Median & IQR & $M^{a}$ & Lower & Upper & \\
\hline \multicolumn{9}{|l|}{ (A) NEI VFQ 25} \\
\hline General health status & 75.00 & 50.00 & 75.00 & 50.00 & 0.00 & 0.00 & 0.00 & 0.705 \\
\hline General vision status & 60.00 & 20.00 & 80.00 & 0.00 & 30.00 & 20.00 & 30.00 & 0.000 \\
\hline Ophthalmic pain & 75.00 & 37.50 & 93.75 & 25.00 & 18.75 & 12.50 & 22.50 & 0.000 \\
\hline Near vision activities & 75.00 & 18.75 & 91.67 & 16.67 & 16.67 & 12.50 & 20.88 & 0.000 \\
\hline Distant vision activities & 66.67 & 27.08 & 91.67 & 16.67 & 20.88 & 16.67 & 25.00 & 0.000 \\
\hline Social functionality & 87.50 & 15.63 & 100.00 & 12.50 & 6.25 & 0.00 & 12.50 & 0.000 \\
\hline Mental health & 62.50 & 31.25 & 87.50 & 14.06 & 21.88 & 15.63 & 28.13 & 0.000 \\
\hline Difficulties in activities & 68.75 & 62.50 & 100.00 & 15.63 & 18.75 & 12.50 & 31.25 & 0.000 \\
\hline Dependence & 91.67 & 27.08 & 100.00 & 0.00 & 8.33 & 4.17 & 16.67 & 0.000 \\
\hline Driving & 66.67 & 29.17 & 91.67 & 12.50 & 25.00 & 16.67 & 29.17 & 0.000 \\
\hline Color vision & 100.00 & 0.00 & 100.00 & 0.00 & 0.00 & 0.00 & 0.00 & 1.000 \\
\hline Peripheral vision & 100.00 & 50.00 & 100.00 & 50.00 & 12.50 & 0.00 & 25.00 & 0.002 \\
\hline \multicolumn{9}{|l|}{ (B) VFQ 39} \\
\hline General health status & 77.50 & -16.25 & 82.50 & -25.00 & 0.00 & 0.00 & 2.50 & 0.091 \\
\hline General vision status & 52.50 & -21.25 & 80.00 & -10.00 & 27.50 & 22.50 & 32.50 & 0.000 \\
\hline Ophthalmic pain & 75.00 & -37.50 & 93.75 & -25.00 & 18.75 & 12.50 & 25.00 & 0.000 \\
\hline Near vision activities & 79.17 & -21.88 & 91.66 & -10.83 & 14.58 & 10.42 & 18.75 & 0.000 \\
\hline Distant vision activities & 70.42 & -20.83 & 91.67 & -12.50 & 20.88 & 16.25 & 24.17 & 0.000 \\
\hline Social functionality & 91.67 & -18.75 & 100.00 & -8.33 & 4.17 & 4.17 & 8.33 & 0.000 \\
\hline Mental health & 65.00 & -35.00 & 90.00 & -16.25 & 22.50 & 15.00 & 30.00 & 0.000 \\
\hline Difficulties in activities & 81.25 & -31.25 & 100.00 & -12.50 & 18.75 & 12.50 & 25.00 & 0.000 \\
\hline Dependence & 93.75 & -20.31 & 100.00 & 0.00 & 6.25 & 3.13 & 12.50 & 0.000 \\
\hline Driving & 66.67 & -29.17 & 91.67 & -12.50 & 25.00 & 16.67 & 29.17 & 0.000 \\
\hline Color vision & 100.00 & 0.00 & 100.00 & 0.00 & 0.00 & 0.00 & 0.00 & 1.000 \\
\hline Peripheral vision & 100.00 & -50.00 & 100.00 & -50.00 & 12.50 & 0.00 & 25.00 & 0.002 \\
\hline \multicolumn{9}{|l|}{ (C) IVI-28 } \\
\hline Mobility and independence & 72.65 & 24.60 & 87.18 & 18.15 & 18.65 & 14.00 & 23.56 & 0.000 \\
\hline Emotional well-being & 63.67 & 15.44 & 79.26 & 22.47 & 20.60 & 15.12 & 26.76 & 0.000 \\
\hline Reading and information awareness & 61.81 & 6.49 & 76.99 & 14.32 & 17.30 & 14.55 & 19.79 & 0.000 \\
\hline
\end{tabular}

Notes: ${ }^{a}$ Estimator Hodges-Lehmann for MD. ${ }^{b} p$-values derived from the Wilcoxon signed-rank test. MD is defined as a median score post-operative - Median Score Preoperative.

Abbreviations: MD, median difference; NEI, National Eye Institute; VFQ, Visual Function Questionnaire.

2. Social functionality and age: correlation coefficient (Spearman) $\rho=0.338(p=0.05)$.

Level of education, spectacle and/or contact lens use, visual acuity, and disease progression do not show any statistically significant correlation with age.

\section{Cronbach's coefficient}

As shown in Table 5A, the Cronbach's coefficient $(\alpha)$ results for VFQ 25 correspond to all parameters but General Health, General Vision, Color Vision, and Peripheral vision, due to their being composed of only one question, as well as the last two scores of VFQ 39 (Table 5B). The coefficient is considered excellent when $\alpha \geq 0.95$, very good if $\alpha \geq 0.8$, and satisfactory if $\alpha \geq 0.7$. The minimum acceptable value is $\alpha \geq 0.6$.

In VFQ 25, pre-operatively the scores of distant vision activities and dependence has unacceptable values and hence are not considered a reliable indicator of QoL evaluation. Post-operatively, the Cronbach's index has satisfactory values in most scores. Reliability issues may exist in the ophthalmic pain score, which was reduced to $\alpha=0.345$ post-operatively. The score of social functionality takes negative value, due to the negative covariance mean value, and violates the reliability hypothesis. 
Table 4 Correlation analysis of age with NEI VFQ 25 and 39 and IVI-28 scores

\begin{tabular}{|c|c|c|c|c|c|c|c|c|}
\hline & \multicolumn{4}{|c|}{ Pre-operative } & \multicolumn{4}{|c|}{ Post-operative } \\
\hline & $\rho$ & $p$-value & $\rho$ & $p$-value & $\rho$ & $p$-value & $\rho$ & $p$-value \\
\hline & \multicolumn{2}{|c|}{ NEI VFQ 25} & \multicolumn{2}{|c|}{ NEI VFQ 39} & \multicolumn{2}{|c|}{ NEI VFQ 25} & \multicolumn{2}{|c|}{ NEI VFQ 39} \\
\hline \multicolumn{9}{|l|}{ (A) NEI VFQ 25 and 39 scores } \\
\hline General health status & -0.199 & 0.260 & -0.180 & 0.310 & -0.116 & 0.514 & -0.102 & 0.566 \\
\hline General vision status & -0.113 & 0.526 & -0.068 & 0.700 & 0.220 & 0.211 & 0.144 & 0.416 \\
\hline Ophthalmic pain & -0.301 & 0.083 & -0.301 & 0.083 & -0.270 & 0.122 & -0.270 & 0.122 \\
\hline Near vision activities & -0.161 & 0.364 & -0.132 & 0.456 & 0.079 & 0.656 & 0.033 & 0.854 \\
\hline Distant vision activities & -0.149 & 0.401 & -0.130 & 0.463 & -0.190 & 0.283 & -0.182 & 0.303 \\
\hline Social functionality & -0.263 & 0.133 & -0.287 & 0.100 & 0.004 & 0.980 & -0.019 & 0.916 \\
\hline Mental health & $-0.397^{*}$ & 0.020 & $-0.354 *$ & 0.040 & -0.197 & 0.265 & -0.206 & 0.244 \\
\hline Difficulties in activities & $-0.389 *$ & 0.023 & $-0.503 * *$ & 0.002 & -0.214 & 0.223 & -0.173 & 0.327 \\
\hline Dependence & -0.093 & 0.602 & -0.094 & 0.599 & -0.005 & 0.979 & -0.007 & 0.969 \\
\hline Driving & -0.260 & 0.210 & -0.260 & 0.210 & -0.272 & 0.189 & -0.272 & 0.189 \\
\hline Color vision & 0.088 & 0.633 & 0.088 & 0.633 & 0.117 & 0.524 & 0.117 & 0.524 \\
\hline Peripheral vision & -0.203 & 0.257 & -0.203 & 0.257 & -0.107 & 0.553 & -0.107 & 0.553 \\
\hline \multicolumn{9}{|l|}{ (B) IVI-28 scores } \\
\hline Mobility and independence & \multicolumn{2}{|l|}{-0.070} & \multicolumn{2}{|l|}{0.701} & \multicolumn{2}{|l|}{-0.030} & \multicolumn{2}{|l|}{0.867} \\
\hline Emotional well-being & \multicolumn{2}{|l|}{-0.267} & \multicolumn{2}{|l|}{0.134} & \multicolumn{2}{|l|}{-0.332} & \multicolumn{2}{|l|}{0.099} \\
\hline Reading and information awareness & \multicolumn{2}{|l|}{-0.335} & \multicolumn{2}{|l|}{0.057} & \multicolumn{2}{|l|}{-0.064} & \multicolumn{2}{|l|}{0.788} \\
\hline
\end{tabular}

Notes: $\rho=$ Spearman coefficient of correlation; $p=$ Wilcoxon Signed Rank Test. *Statistically significant for 0.05 (two-tailed). **Statistically significant for 0.01 (two-tailed). Abbreviations: NEI, National Eye Institute; VFQ, Visual Function Questionnaire.

VFQ 39 demonstrates better reliability. Specifically, the negative value of the coefficient for the social functionality score is eliminated, but still has a non-acceptable value. Pre-operatively, the index for dependency is marginally non-acceptable, whereas the remaining eight sets of scores are reliable for data evaluation. Post-operatively, there are negative index values, which in general health score moves to borderline non-acceptable value, whereas in the ophthalmic pain score is well below the minimum acceptable threshold.

The IVI questionnaire demonstrated very high-reliability coefficient both before and after surgery (Table 5C). However, it should be noted that the large number of questions within each group contributes to the increased coefficient values.

\section{Discussion}

$\mathrm{KCN}$ is an idiopathic degenerative eye disease characterized by localized thinning and conical protrusion of the cornea. Progressive, asymmetrical corneal steepening associated with an increase in myopic and astigmatic refractive errors, combined with midperipheral and/or peripheral corneal thinning represent some of the findings associated with this ectatic corneal disorder. It affects young individuals and progression occurs until 30-40 years of age. ${ }^{23}$

$\mathrm{KCN}$ is currently managed using a number of methods. In early stages, spectacles or soft contact lenses can correct refractive errors sufficiently. As the disease progresses, these methods become unsuitable for correcting the high levels of irregularity associated with $\mathrm{KCN}$. For advanced stages of $\mathrm{KCN}$, rigid gas permeable (RGP) contact lenses have become the most common and successful method of management providing improved visual acuity while decreasing the need for surgical interventions. In the case of RGP intolerance, or in advancing keratectasia progression, a penetrating keratoplasty (PK) is used to replace the entire cornea with healthy donor tissue. ${ }^{24}$ However, previous research has shown that despite satisfactory results on visual outcome measures obtained after PK, vision-related QoL in KCN patients remains impaired. ${ }^{25,26}$ Furthermore, intracorneal ring segment (ICRS) implantation is a surgical option to treat $\mathrm{KCN}$, which avoids corneal graft and which showed that visual QoL remained good over 1 year post-operatively. ${ }^{27}$

In recent years, the combination of CXL with excimer laser partial photorefractive keratectomy (PRK), known 
Table 5 Cronbach's index results

\begin{tabular}{|c|c|c|c|}
\hline & & $\begin{array}{l}\text { Pre- } \\
\text { operative }\end{array}$ & $\begin{array}{l}\text { Post- } \\
\text { operative }\end{array}$ \\
\hline & $\mathbf{N}^{\mathbf{a}}$ & $\alpha$ & $\alpha$ \\
\hline \multicolumn{4}{|c|}{ (A) Cronbach's index for NEI VFQ 25 pre- and post-operativ } \\
\hline Ophthalmic pain & 2 & 0.600 & 0.345 \\
\hline Near vision activities & 3 & 0.680 & 0.715 \\
\hline Distant vision activities & 3 & 0.589 & 0.679 \\
\hline Social functionality & 2 & -0.040 & -0.360 \\
\hline Mental health & 4 & 0.600 & 0.798 \\
\hline Difficulties in activities & 2 & 0.889 & 0.925 \\
\hline Dependence & 3 & 0.551 & 0.746 \\
\hline Driving & 3 & 0.824 & 0.809 \\
\hline \multicolumn{4}{|c|}{ (B) Cronbach's index for NEI VFQ 39 pre- and post-operativ } \\
\hline General health status & 2 & 0.636 & 0.584 \\
\hline General vision status & 2 & 0.851 & $0.74 I$ \\
\hline Ophthalmic pain & 2 & 0.600 & 0.345 \\
\hline Near vision activities & 6 & 0.783 & 0.807 \\
\hline Distant vision activities & 6 & 0.737 & 0.798 \\
\hline Social functionality & 3 & 0.469 & 0.144 \\
\hline Mental health & 5 & 0.727 & 0.847 \\
\hline Difficulties in activities & 4 & 0.831 & 0.913 \\
\hline Dependence & 4 & 0.531 & 0.720 \\
\hline Driving & 3 & 0.824 & 0.809 \\
\hline \multicolumn{4}{|c|}{ (C) Cronbach's index for IVI-28 scores pre- and post-operative } \\
\hline Mobility and independence & 11 & 0.843 & 0.897 \\
\hline Emotional well-being & 8 & 0.847 & 0.930 \\
\hline $\begin{array}{l}\text { Reading and information } \\
\text { awareness }\end{array}$ & 9 & 0.859 & 0.815 \\
\hline
\end{tabular}

Note: ${ }^{\mathrm{N}} \mathrm{N}=$ number of questions within each group.

Abbreviations: NEI, National Eye Institute; VFQ, Visual Function Questionnaire.

today as the Athens Protocol, ${ }^{28,29}$ has demonstrated potential for retarding or eliminating the progression of $\mathrm{KCN} .{ }^{30-33}$ However, for this combined procedure, several studies have been conducted showing satisfactory clinical results without ${ }^{34-38}$ taking into consideration the QoL change and response shift of patients. Bearing in mind the aforementioned data, for the purpose of the current study, the NEI VFQ and the IVI questionnaire were used.

The results of this study, derived from non-parametric tests, indicate a clear improvement in QoL following the Athens Protocol procedure. It is notable that post-operatively not only all the scores show statistical significance but also the values of $p$ are identical, except those of general health and overall vision. In NEI VFQ 25/39, all scores show remarkable improvement, other than those of general health and color vision, which do not affect the $\mathrm{QoL}$ in patients with $\mathrm{KCN}$. The scores of the general vision, mental health, and driving ability showed the greatest improvement, whereas the scores of distant and near vision activities, the difficulties of the activity, and the ophthalmic pain showed measurable improvement. The importance of mental health is confirmed by the results of the IVI-28 questionnaire in which the score of emotional wellness represents the greatest improvement, as compared to the level of mobility and independence and the scores of reading and information awareness. Mental health pre-operatively is also significantly affected by gender based on the NEI VFQ 25. In addition to this, Fink et al ${ }^{38}$ reported that gender differences exist in patient history, vision, and ocular symptoms in $\mathrm{KCN}$ patients.

The VFQ 25/39 results appear to be influenced by age. Younger age patients tend to be more positive about their state of mental health and difficulties in their activities prior to the operation. Although this finding is not supported by the existing literature, it could be attributed to the stage of the disease, which is usually early or mild compared to older age patients. Also, as implied by the investigation, the resulting difference between the post-operative and pre-operative status of patients on the scale of "difficulty activities" and "social functionality" of NEI VFQ 39 is partly due to age. The fact that higher age of respondents results in greater score difference is perhaps due to the stage of the disease. However, it should again be noted that due to the originality of research, there are no such references, and therefore this specific hypothesis is based on individual clinical data.

This study is subject to several limitations. First, an obvious limitation of our study is the fact that we included some cases where both eyes were treated and some cases where just one eye was treated. Although the majority of cases were binocular treatments, we should note here that all treatments were performed on patients who were contact lens intolerant and showed significant visual debilitation. Thus, on this ground, we can consider that either treating one eye or treating both eyes was a measure taken to improve the visual function and the QoL of these patients, and thus with this token they should be viewed as similar interventions. Second, perhaps more recent questionnaires could be more accurate. Finally, some people tend to accommodate to their health stage; QoL, therefore, may show some improvement through time even without intervention. Our data therefore should be viewed with this possible consideration in mind as well.

\section{Conclusion}

The findings of this study indicate a clear subjectively assessed improvement in QoL in keratoconic patients 
subjected to the Athens Protocol procedure. This positive change stems from the improvement of all their interdependent clinical and psychological features. Reducing irregular astigmatism, increasing visual acuity, and inhibiting the progression of KCN demonstrate significant improvement in activities requiring distant vision and near vision, such as driving ability, reading, and receiving information. The IVI-28 questionnaire proved reliable for the purposes of this research, although reliability issues arose mainly for social functioning score of NEI VFQ.

\section{Disclosure}

Dr Anastasios John Kanellopoulos is a consultant for Alcon; Allergan; Avedro; i-Optics; Keramed; Zeiss; ISP Surgical. The author reports no other conflicts of interest in this work.

\section{References}

1. Veenhoven R. Quality of life research. In: Bryant CD, Peck DL, editors. 1st Century Sociology, a Reference Handbook. Vol. 2. Chapter 7. Thousand Oaks, CA: Sage; 2007:54-62.

2. Haas BK. A multidisciplinary concept analysis of quality of life. West J Nurs Res. 1999;21:728-742. doi:10.1177/01939459922044153

3. Diener E, Suh E. Measuring quality of life: economic, social, and subjective indicators. Soc Indic Res Kluwer Acad Publ. 1997;40:189-216. doi:10.1023/A:1006859511756

4. Calman KC. Quality of life in cancer patients - an hypothesis. J Med Ethics. 1984;10:124-127.

5. Folland S, Goodman AC, Stano M. The Economics of Health and Health Care. 7th ed. New Jersey: Pearson Education Inc.; 2013:77-94, 295.

6. Gill TM, Feinstein AR. A critical appraisal of the quality of quality-oflife measurements. JAMA. 1994;272:619-626.

7. Bowling A. Mode of questionnaire administration can have serious effects on data quality. J Public Health (Oxf). 2005;27:281-291. doi:10. 1093/pubmed/fdi031

8. Sibbald B, Addington-Hall J, Brenneman D, Freeling P. Telephone versus postal surveys of general practitioners: methodological considerations. Br J Gen Pract. 1994;44:297-300.

9. Fayers MP, Machin D. Quality of Life: Assessment Analysis and Interpretation. West Sussex: John Wiley \& Sons Ltd; 2000:45-71.

10. Margolis MK, CoyneK, Kennedy-Martin T, BakerT, Schein O, RevickiDA. Vision-specific instruments for the assessment of health-related quality of life and visual functioning: a literature review. Pharmacoeconomics. 2002;20:791-812. doi:10.2165/00019053-200220120-00001

11. Kymes SM, Walline JJ, Zadnik K, Gordon MO. Collaborative longitudinal evaluation of keratoconus study group. Quality of life in keratoconus. Am J Ophthalmol. 2004;138:527-535. doi:10.1016/j.ajo.2004.04.031

12. Gothwal VK, Reddy SP, Fathima A, et al. Assessment of the impact of keratoconus on vision-related quality of life. Invest Ophthalmol Vis Sci. 2013;54:2902-2910. doi:10.1167/iovs.12-10783

13. Khadka J, Schoneveld PG, Pesudovs K. Development of a keratoconusspecific questionnaire using Rasch analysis. Optom Vis Sci. 2017;94(3): 395-403. doi:10.1097/OPX.0000000000001035

14. Labiris G, Giarmoukakis A, Sideroudi H, Gkika M, Fanariotis M, Kozobolis V. Impact of keratoconus, cross-linking and cross-linking combined with photorefractive keratectomy on self-reported quality of life. Cornea. 2012;31:734-739. doi:10.1097/ICO.0b013e31823cbe85

15. Mangione CM, Lee PP, Gutierrez PR, Spritzer K, Berry S, Hays RD; National Eye Institute Visual Function Questionnaire Field Test Investigators. Development of the 25-item National Eye Institute Visual Function Questionnaire. Arch Ophthalmol. 2001;119:1050-1058.
16. Visual Function Questionnaire (VFQ-25). Available from: https:// www. rand.org/health/surveys_tools/vfq.html. Accessed October 10, 2015." To "Carol M. Mangione, Paul P. Lee, Peter R. Gutierrez et al Development of the 25-list-item National Eye Institute Visual Function Questionnaire. Arch Ophthalmol. 2001;119(7):1050-1058.

17. Goulia O, Karampataki V, Kandili D. National Eye Institute Visual Functioning Questionnaire-25 - Greek. School of Medicine Aristotle University of Thessaloniki, Greece. Version 2000.

18. Weih LM, Hassell JB, Keeffe J. Assessment of the impact of vision impairment. Invest Ophthalmol Vis Sci. 2002;43:927-935.

19. Finger RP, Tellis B, Crewe J, Keeffe JE, Ayton LN, Guymer RH. Developing the Impact of Vision Impairment-Very Low Vision (IVI-VLV) questionnaire as part of the LoVADA protocol. Invest Ophthalmol Vis Sci. 2014;55(4):6150-6158. doi:10.1167/iovs.14-14731

20. Lamoureux EL, Pallant JF, Pesudovs K, Rees G, Hassell JB, Keeffe JE. The impact of vision impairment questionnaire: an assessment of its domain structure using confirmatory factor analysis and Rasch analysis. Invest Ophthalmol Vis Sci. 2007;48:1001-1006. doi:10.1167/iovs.06-0361

21. Karabatsas CH, Cook SD, Sparrow JM. Proposed classification for topographic patterns seen after penetrating keratoplasty. Br J Ophthalmol. 1999;83:403-409.

22. Rabinowitz YS. Keratoconus. Surv Ophthalmol. 1998;42:297-319.

23. Gore DM, Shortt AJ, Allan BD. New clinical pathways for keratoconus. Eye (Lond). 2013;27:329-339. doi:10.1038/eye.2012.257

24. Yildiz EH, Cohen EJ, Virdi AS, Hammersmith KM, Laibson PR, Rapuano CJ. Contact lens related quality of life in patients with keratoconus. Eye Contact Lens. 2009;35:123-127. doi:10.1097/ICL. 0b013e31819d1dd0

25. Yildiz EH, Erdurmus M, Savran Elibol E, Acar B, Vural ET. Contact lens impact on quality of life in keratoconus patients: rigid gas permeable versus soft silicone-hydrogel keratoconus lenses. Int J Ophthalmol. 2015;8:1074-1077.

26. Freitas Santos Paranhos J, Avila MP, Paranhos A Jr, Schor P. Visual perception changes and optical stability after intracorneal ring segment implantation: comparison between 3 months and 1 year after surgery. Clin Ophthalmol. 2011;5:1057-1062. doi:10.2147/OPTH.S23147

27. Kanellopoulos AJ. Long-term results of a prospective randomized bilateral eye comparison trial of higher fluence, shorter duration ultraviolet A radiation, and riboflavin collagen cross linking for progressive keratoconus. Clin Ophthalmol. 2012;6:97-101. doi:10.2147/OPTH.S27170

28. Kanellopoulos AJ, Asimellis G. Keratoconus management: longterm stability of topography-guided normalization combined with high-fluence CXL stabilization (the Athens Protocol). J Refract Surg. 2014;30:88-93. doi:10.3928/1081597X-20140120-03

29. Wollensak G, Spoerl E, Seiler T. Riboflavin/ultraviolet-a-induced collagen crosslinking for the treatment of keratoconus. Am J Ophthalmol. 2003;135:620-627.

30. Vinciguerra P, Albé E, Frueh BE, Trazza S, Epstein D. Two-year corneal cross-linking results in patients younger than 18 years with documented progressive keratoconus. Am J Ophthalmol. 2012;154:520-526. doi:10.1016/j.ajo.2012.03.020

31. Caporossi A, Mazzotta C, Baiocchi S, Caporossi T. Long-term results of riboflavin ultraviolet a corneal collagen cross-linking for keratoconus in Italy: the Siena eye cross study. Am J Ophthalmol. 2010;149:585-593. doi:10.1016/j.ajo.2009.10.021

32. O'Brart DPS, Kwong TQ, Patel P, et al. Long-term follow-up of riboflavin/ultraviolet A $(370 \mathrm{~nm})$ corneal collagen cross-linking to halt the progression of keratoconus British. JOphthalmol. 2013;97:433-437.

33. Krueger RR, Kanellopoulos AJ. Stability of simultaneous topographyguided photorefractive keratectomy and riboflavin/UVA crosslinking for progressive keratoconus: case reports. J Refract Surg. 2010;26:S827-S832. doi:10.3928/1081597X-20100921-11

34. Kanellopoulos AJ, Asimellis G. Introduction of quantitative and qualitative cornea optical coherence tomography findings, induced by collagen cross-linking for keratoconus; a novel effect measurement benchmark. Clin Ophthalmol. 2013;7:329. doi:10.2147/OPTH.S40455 
35. Markakis GA, Roberts CJ, Harris JW, Lembach RG. Comparison of topographic technologies in anterior surface mapping of keratoconus using two display algorithms and six corneal topography devices. Int $J$ of Kerat and Ectatic Dis. 2012;1:153-157.

36. Sakla H, Altroudi W, Muñoz G, Albarrán-Diego C. Simultaneous topography-guided partial photorefractive keratectomy and corneal collagen crosslinking for keratoconus. J Cataract Refract Surg. 2014;40: 1430-1438. doi:10.1016/j.jcrs.2013.12.017
37. Spadea L, Paroli M. Simultaneous topography-guided PRK followed by corneal collagen cross-linking after lamellar keratoplasty for keratoconus Clin Ophthalmol (Auckland, NZ). 2012;6:1793-1800. doi:10.2147/OPTH

38. Fink BA, Wanger H, Steger-May K, et al. Differences in keratoconus as a function of gender. Am J Ophthalmol. 2005;140:469-e1. doi:10. 1016/j.ajo.2005.03.078

\section{Publish your work in this journal}

Clinical Ophthalmology is an international, peer-reviewed journal covering all subspecialties within ophthalmology. Key topics include: Optometry; Visual science; Pharmacology and drug therapy in eye diseases; Basic Sciences; Primary and Secondary eye care; Patien Safety and Quality of Care Improvements. This journal is indexed on

Submit your manuscript here: http://www.dovepress.com/clinical-ophthalmology-journal

\section{Dovepress}

PubMed Central and CAS, and is the official journal of The Society of Clinical Ophthalmology (SCO). The manuscript management system is completely online and includes a very quick and fair peer-review system, which is all easy to use. Visit http://www.dovepress.com/ testimonials.php to read real quotes from published authors. 